\section{Antenatal diagnosis of critical congenital heart disease. Optimal place of delivery is where appropriate care can be delivered}

\author{
Julene S Carvalho 1,2,3
}

Congenital heart disease (CHD) is considered major if it requires cardiac surgery or catheter intervention or results in death in the first year of life, while it is defined as critical if these occur in the first 28 days. Current strategies to increase early diagnosis of such defects involve improving antenatal screening programmes and implementing neonatal screening by pulse oximetry. Structural forms of critical CHD are usually associated with a duct-dependent lesion. Within this group, the spectrum of outcome varies, from guarded outlook such as in hypoplastic left heart syndrome (HLHS) to conditions with better outcome such as complete transposition of the great arteries (TGA).

Eckersley and colleagues ${ }^{1}$ report the results of a population-based study carried out in New Zealand (2006-2010), where one in five neonates with critical CHD had late diagnosis (after discharge from hospital following birth or at postmortem examination), while the remainder were diagnosed early (prenatally or before discharge). For these potentially critically ill infants, the authors showed an impact of timing of diagnosis (early vs late) on 1-year mortality. Such an impact was more significant in isolated critical CHD (early diagnosis; 12\%, 95\% CI 9\% to $17 \%$ vs late diagnosis; $29 \%$, 95\% CI $20 \%$ to $41 \%$ ), highlighting the role of associated syndromes and major extracardiac abnormalities on overall infant mortality. It follows that for critical CHD, isolated or not, early diagnosis is better and every effort should be made to achieve this goal. However, despite being diagnosed early, some neonates may still die if prompt cardiac intervention is not

\footnotetext{
${ }^{1}$ Brompton Centre for Fetal Cardiology, Royal Brompton Hospital NHS Foundation Trust, London, UK; ${ }^{2}$ Fetal Medicine Unit, St George's University Hospital NHS Foundation Trust, London, UK; ${ }^{3}$ Institute of Cardiovascular and Cell Sciences. St George's, University of London, London, UK

Correspondence to Dr Julene S Carvalho, Brompton Centre for Fetal Cardiology, Royal Brompton Hospital, Sydney Street, London SW3 6NP, UK; j.carvalho@rbht. nhs.uk
}

available. In the accompanying study, ${ }^{1}$ neonatal deaths in complete TGA were mainly related to distance between place of birth and the cardiac centre. Eckersley and colleagues ${ }^{1}$ highlight the importance of time of diagnosis. Place of delivery will further influence outcome in critical CHD.

Similarly, in a recent meta-analysis, Holland $e t a l^{2}$ showed that prenatal diagnosis of critical CHD improved preoperative neonatal survival (pooled OR 0.26; 95\% CI, 0.08 to 0.84 ) when comparing cases with similar forms of $\mathrm{CHD}$, standard risk and intention to treat. Better survival could only be demonstrated if fetal cases followed an optimal delivery plan and after excluding death of a neonate with fetal diagnosis of TGA who was delivered outside a centre with facilities for balloon atrial septostomy.

Thus, place of delivery should be taken into account when a perinatal management plan is put in place for fetuses with critical CHD. The question is: should all such babies be delivered at or in close proximity to tertiary centres or can they be delivered in the local hospital? To put this in perspective, it is important to recognise critical abnormalities that may require urgent catheter or surgical intervention shortly after birth and thus benefit from delivery at a specialised centre (table 1).

\section{CRITICAL CHD EXPECTED TO BE HAEMODYNAMICALLY STABLE AND NOT TO REQUIRE URGENT} POSTNATAL INTERVENTION

Arterial duct-dependent circulation

In general, critical forms of CHD are duct dependent, be it pulmonary or systemic circulation. These can be stabilised postnatally by elective intravenous infusion of prostaglandin E (table 1). Examples include left-sided obstructive lesions such as coarctation of the aorta and standard risk HLHS, right-sided lesions such as pulmonary atresia with or without a ventricular septal defect but also complex CHD (eg, atrial isomerism, double inlet ventricle and tricuspid atresia) associated with critical obstruction to pulmonary or systemic flow. Therefore, site of delivery requires a hospital where this level of neonatal care can be provided safely, including facilities for mechanical ventilation. This management care plan is supported by the retrospective study of Bennett et $a l^{3}$ showing no significant difference in 90-day mortality between infants with duct-dependent lesions delivered in specialised centres or other centres.

In summary, for most duct-dependent lesions, close proximity to a cardiac centre is not necessary, provided the neonate can be stabilised safely in the local hospital and subsequently transferred electively to the cardiac centre. ${ }^{4}$

\section{CRITICAL CHD EXPECTED TO REQUIRE URGENT POSTNATAL INTERVENTION}

CHD with foramen ovale-dependent circulation

The above publications ${ }^{1} 2$ support further the current approach that pregnancies with fetal diagnosis of simple TGA should have obstetric care optimised to allow for urgent balloon atrial septostomy, if needed. The sensitivity of fetal echocardiography to predict the need for urgent intervention is low (table 1). However, in cases where the atrial septum appears restrictive prenatally, a multidisciplinary team involving an interventional paediatric cardiologist should be at or near the delivery room.

The same reasoning can be extrapolated to other forms of CHD whereby a restrictive interatrial communication is likely to compromise neonatal haemodynamic stability. Typically, this occurs in HLHS with highly restrictive/intact atrial septum (table 1). Other forms of complex CHD may also depend on the adequacy of flow across the atrial septum. If it is restrictive prenatally, delivery near a cardiac centre is recommended.

CHD with obstructed pulmonary venous return/ductus venosus-dependent circulation

Isolated total anomalous pulmonary venous connection is uncommon and rarely diagnosed prenatally, so that choosing place of delivery may not be an option. However, it is commonly seen in complex CHD associated with right isomerism, which is often detected prenatally. Isolated or not, pulmonary venous obstruction can be supracardiac or infracardiac and while the obstruction may be reasonably well tolerated prenatally, the neonate is expected to develop pulmonary oedema soon after changes in the perinatal circulation, which can be aggravated 
Table 1 Suggested delivery options for infants with prenatally diagnosed critical CHD

\begin{tabular}{|c|c|c|}
\hline Critical CHD & Comments & Expected neonatal cardiovascular status \\
\hline $\begin{array}{l}\text { Duct-dependent pulmonary } \\
\text { circulation } \\
\text { All forms of CHD requiring PGE to } \\
\text { maintain pulmonary blood flow }\end{array}$ & $\begin{array}{l}\text { Includes all forms of CHD associated } \\
\text { with: } \\
\text { pulmonary atresia* or } \\
\text { - severe pulmonary stenosis }\end{array}$ & $\begin{array}{l}\text { Without additional critical lesion } \\
\text { Stable neonate on PGE infusion }\end{array}$ \\
\hline
\end{tabular}

$\begin{array}{ll}\text { Duct-dependent systemic } & \text { Includes all forms of CHD associated } \\ \text { circulation } & \text { with: } \\ \text { All forms of CHD requiring PGE to } & \text { aortic atresia or } \\ \text { maintain systemic blood flow } & \text { severe aortic stenosis or } \\ & - \text { aortic coarctationt or } \\ & \text { aortic interruption }\end{array}$

Complete transposition

No major associated abnormalities

Other foramen ovale-dependent circulation

TAPVC, obstructed

CHD associated with important valvar regurgitation and/or myocardial dysfunction
CHD that may require balloon atrial septostomy

Includes CHD associated with mitral or tricuspid atresia or severe stenosis

Isolated TAPVC or associated with complex lesions (eg, isomerism)

- Ebstein malformation or dysplasia of the tricuspid valve

- Truncus arteriosus with severe truncal valve regurgitation

- Tetralogy of Fallot with absent pulmonary valve syndrome with severe pulmonary regurgitation

- CHD with severe atrioventricular valve regurgitation
With additional critical lesion, that is, neonate is expected to require urgent intervention

- Obstructed pulmonary venous return in complex cases associated with right isomerism

- Restrictive atrial septum in cases with associated mitral/tricuspid atresia or severe stenosis

- Potentially unstable neonate

Without additional critical lesion

- Stable neonate on PGE infusion

With additional critical lesion, that is, neonate is expected to require urgent intervention

- HLHS with highly restrictive/intact atrial septum

- Restrictive atrial septum in cases with associated mitral or tricuspid atresia or severe stenosis

- Potentially unstable neonate

Potentially unstable neonate

Atrial septum can become restrictive shortly after birth (low sensitivity of prenatal scans)

Fetus at risk of postnatal restriction of foramen ovale if septum primum bulges $>50 \%$ towards the left atrium, forms an angle $<30^{\circ}$ with the rest of the septum or does not swing with the cardiac cycle

Highly unstable neonate if the arterial duct is also constricted

Potentially unstable neonate if atrial septum is severely restrictive

In HLHS and mitral atresia, a 'to-and-fro' pattern of pulmonary venous Doppler signal indicates severely restricted/intact atrial septum

Potentially unstable neonate due to obstruction at different sites

Potentially unstable neonate

(1)

Place of delivery and care plan

Can be delivered in local hospital, with facilities to stabilise the neonate

- PGE infusion

- Transfer to cardiac centre Planned delivery at or near cardiac centre with surgical and catheter interventional facilities

Can be delivered in local hospital, with facilities to stabilise the neonate

- PGE infusion

- Transfer to cardiac centre Planned delivery at or near cardiac centre with surgical and catheter interventional facilities

Planned delivery at or near cardiac centre with facilities for balloon atrial septostomy

Planned delivery at or near cardiac centre with facilities for balloon atrial septostomy

Planned delivery at or near cardiac centre with surgical and catheter interventional facilities

Planned delivery at tertiary neonatal unit or near cardiac centre

\footnotetext{
${ }^{*}$ Cases with pulmonary atresia with VSD and collaterals are usually non-critical lesions.

†For cases of suspected coarctation of the aorta, the elective use of PGE will depend on the degree of suspicion as antenatal diagnosis has a relatively high false-positive rate.

CHD, congenital heart disease; HLHS, hypoplastic left heart syndrome; PGE, prostaglandin E; TAPVC, total anomalous pulmonary venous connection; VSD, ventricular septal defect.
}

by postnatal closure of the ductus venosus in the infradiaphragmatic type. Thus, to optimise neonatal outcome, delivery should also take place at close proximity to a surgical centre, as urgent cardiac surgery is required.

\section{CRITICAL CHD ASSOCIATED WITH SEVERE VALVAR REGURGITATION AND/OR CARDIOMEGALY}

The fetus with critical CHD and severe atrioventricular or semilunar regurgitation leading to cardiomegaly may remain haemodynamically stable in fetal life but may also develop hydrops. In either case, the neonate is likely to be unstable. Prediction of postnatal level of care required to stabilise the neonate with cardiomegaly with or without myocardial dysfunction may vary from case to case. Risk assessment should take into account any concerns regarding possible lung hypoplasia, the presence of fetal hydrops or any other indicator of potential neonatal instability. Such fetuses are unlikely to require surgery in the first few hours of life, but will require high level of intensive care, so delivery should be planned in hospitals with tertiary neonatal units or near a cardiac centre.

\section{CRITICAL CHD-OTHER FACTORS THAT MAY AFFECT PLACE OF DELIVERY}

One of the main factors that influences place of delivery is spontaneous onset of labour leading to prematurity, which is often unpredictable. Thus, despite having a careful perinatal management plan tailored for the individual fetus, delivery may occur unexpectedly at a different location. In some instances, however, premature labour may be anticipated such as in cases with significant polyhydramnios due to associated gastrointestinal obstruction. It is therefore important that a perinatal management plan be defined early in pregnancy, 
documented in the pregnant woman's notes and updated as pregnancy advances, according to the ultrasound findings.

The presence of additional major extracardiac abnormalities is another factor that may dictate place of delivery and should be taken into account in all forms of critical CHD.

\section{CONCLUSIONS}

Place of delivery of the fetus with critical CHD should be decided based on individual diagnosis and assessment of risk associated with the cardiac diagnosis and its pathophysiology, as well as the level of care available locally. ${ }^{5}$ Decision making should be tailored to the individual case, taking all factors into account-but ensuring that place of elective delivery is optimal and sufficiently placed to look after the neonate, with the awareness that a significant number of babies will be born prematurely. The best strategy therefore is to define a clear perinatal management plan and good communication with local teams. Time of delivery (and therefore place of delivery) may be unpredictable. Ultimately however, the best place to deliver the fetus with critical CHD is where appropriate care can be delivered safely.

\section{Competing interests None declared.}

Provenance and peer review Commissioned; internally peer reviewed.

To cite Carvalho JS. Arch Dis Child 2016;101:505507.

Received 6 July 2015

Revised 25 December 2015

Accepted 28 December 2015

Published Online First 27 January 2016

\section{(5) Linked}

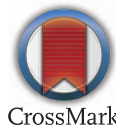

http://dx.doi.org/10.1136/archdischild-2014-307691
Arch Dis Child 2016;101:505-507.

doi:10.1136/archdischild-2015-308736

\section{REFERENCES}

1 Eckersley L, Saddler L, Parry E, et al. Timing of diagnosis affects mortality in critical congenital heart disease. Arch Dis Child 2016:101:514-8.

2 Holland BJ, Myers JA, Woods CR Jr. Prenatal diagnosis of critical congenital heart disease reduces risk of death from cardiovascular compromise prior to planned neonatal cardiac surgery: a meta-analysis. Ultrasound Obstet Gynecol 2015;45:631-8.

3 Bennett TD, Klein MB, Sorensen MD, et al. Influence of birth hospital on outcomes of ductal-dependent cardiac lesions. Pediatrics 2010;126:1156-64.

4 Bartsota M, Judd N, Carvalho JS. When, how and where to deliver the fetus with major congenital heart disease. Fetal Matern Med Rev 2014;25:79-94.

5 Donofrio MT, Levy RJ, Schuette JJ, et al. Specialized delivery room planning for fetuses with critical congenital heart disease. Am J Cardiol 2013:111:737-47. 